\title{
Características dos cursos voltados para a formação em gestão em saúde no Brasil
}

\author{
Characteristics of health management courses in Brazil
}

Maria Luiza Silva Cunha1', Virginia Alonso Hortale²

RESUMO O objetivo do artigo foi discutir as características da formação em gestão em saúde oferecida no Brasil, desde sua origem histórica e de oferta de cursos. O estudo foi realizado por meio de um mapeamento de cursos de gestão em saúde, de diferentes níveis de formação, levantados em sítios institucionais. Os cursos se caracterizam pela diversidade em sua denominação, modalidade, categoria administrativa, região e ano de início. O enfrentamento do desafio de gestão do Sistema Único de Saúde (SUS) guarda relação com iniciativas de formação de seus gestores. O direcionamento à área hospitalar na graduação e na especialização, com predomínio do setor privado, pode ser um limite ao modelo de atenção previsto no SUS.

PALAVRAS-CHAVE Desenvolvimento de pessoal. Cursos. Gestão em saúde. Sistema Único de Saúde.

ABSTRACT This article's objective was to discuss the characteristics of health management training offered in Brazil, from its historical origin and the supply of courses. The study involved mapping the country's health management courses at different levels of training, identified on institutional sites. The courses display a wide diversity in their nomenclature and other aspects. Facing the challenge of managing the Unified Health System (SUS) relates to the initiatives for training its administrators. The focus on the hospital area in undergraduate training and in specialization courses, predominantly in the private sector, may be a limitation for the intended model of care in the SUS.

1 Fundação Oswaldo Cruz (Fiocruz), Escola Nacional de Saúde Pública Sergio Arouca (Ensp), Programa de Pós-Graduação em Saúde Pública e Escola Politécnica de Saúde Joaquim Venâncio (EPSJV), Laboratório de Educação Profissional em Gestão em Saúde - Rio de Janeiro (RJ), Brasil. luizacunha@fiocruz.br

2 Fundação Oswaldo Cruz (Fiocruz), Escola Nacional de Saúde Pública Sergio Arouca (Ensp) - Rio de Janeiro (RJ), Brasil.

virginia@ensp.fiocruz.br 


\section{Introdução}

No presente estudo, cujo objeto é a formação em gestão em saúde no Brasil, partiu-se do interesse em discutir as características dessa formação desde sua origem histórica e de oferta de cursos. Tomou-se como base a constatação de que o contexto político-econômico mais amplo apresentou reflexos não apenas na implementação do Sistema Único de Saúde (SUS), mas igualmente na formação dos gestores responsáveis pela sua operacionalização.

Nos últimos anos, a gestão em saúde vem sendo apontada como um dos grandes desafios para a consolidação do SUS. Campos (2006), ao discorrer sobre a gestão de serviços de saúde, chama a atenção para a relação de dependência entre a mesma e a política hegemônica em um dado período. Na medida em que essa se volta ao mercado, predomina o modelo de gestão direcionado aos interesses privados, com decisões que atendem às corporações, de médicos, de outros profissionais ou de empresários, em detrimento dos interesses e necessidades de saúde da população.

Para Rodrigues (2014), as dificuldades da gestão encontram-se associadas ao debilitamento proposital da burocracia pública, em um contexto de predomínio da política neoliberal, desenvolvida de forma contraditória com a adoção do modelo social-democrata e com as políticas de proteção social inscritas na Constituição Federal de 1988.

Com a implantação do SUS em 1988, o modelo de atenção à saúde proposto voltava-se não somente ao desenvolvimento de ações preventivas, mas à integralidade, equidade e universalidade das ações. Tais mudanças não possuíam correspondência, entretanto, com os programas de formação de gestores, que apresentavam limites como a falta de definição do profissional adequado, do desenho curricular e da metodologia, da inexistência de docentes preparados para esta formação, entre outros (VECINA NETO, 1990).

A descentralização, que tomou os municípios como centro da política, significou, para
Silva (2005), a preocupação com o aumento da capacidade de gerenciamento do sistema de saúde e com a formação de profissionais para fazer frente aos novos desafios na condução da política de saúde.

Estudo de Machado, Lima e Baptista (2007) destaca que a opção pela descentralização da gestão do sistema de saúde para os municípios, adotada no SUS, envolveu mudanças nas relações intergovernamentais, com alterações significativas no que concerne às atribuições dos gestores desse setor. Além disso, no que se refere ao nível local, a heterogeneidade da conformação dos municípios brasileiros implicaria diferentes capacidades de ordem política, institucional e econômica por parte dos gestores municipais.

Pierantoni (2006), no seu trabalho sobre o Mestrado Profissional em Administração de Saúde, afirma que os sistemas e serviços de saúde possuem especificidades e requerem a aplicação de diferentes técnicas com base em um referencial teórico-prático das áreas de administração e planejamento em saúde. Nesse sentido, não poderia ser considerada uma variante da 'administração de negócios'. A formação na área:

[...] apresenta uma precariedade do ensino da administração e organização dos serviços de saúde, em diferentes cursos de graduação, tanto na área das ciências sociais e da saúde quanto em outros setores do conhecimento. (PIERANTONI, 2006, P. 125).

Em decorrência, gestores e profissionais vinculados ao mercado de trabalho de prestação de serviços de saúde passaram a buscar os cursos de pós-graduação em saúde coletiva, seja acadêmico ou profissional. De acordo com Kisil (1994), no Brasil, assim como em outros países da América Latina, o ensino da denominada administração em saúde esteve ligado na sua origem aos cursos de formação de sanitaristas.

Ao se considerar a importância da gestão no pleno desenvolvimento do SUS e, consequentemente, da formação dos gestores que 
operam as práticas de saúde no cotidiano dos sistemas e serviços, interroga-se sobre quais eram as características dessa formação, com base em aspectos históricos e de oferta de cursos. Assim, desenvolveu-se o presente estudo em dois eixos: no primeiro, buscou-se conhecer elementos históricos da política de formação na área e a sua correspondência com a oferta nacional de cursos de gestão em saúde, destacando o cenário, os objetivos e os agentes envolvidos; no segundo, apresentam-se as principais características da formação em gestão em saúde no Brasil, no que se refere às suas diferentes denominações, modalidade, categoria administrativa das instituições ofertantes, distribuição geográfica, carga horária, grau acadêmico e ano de início. Ao final, discorre-se sobre os avanços, desafios e perspectivas futuras da formação em gestão em saúde no Brasil.

\section{Métodos}

O estudo desenvolveu-se em duas etapas. Uma primeira, em que se realizou uma revisão não extensiva da literatura documental e científica. Adotaram-se, como critério de escolha, estudos cujo foco eram os componentes da história do SUS e da formação em saúde, publicados entre os anos 1988 a 2015, utilizando os termos 'educação' and 'gestão em saúde', cadastrados nos Descritores em Ciências da Saúde (DeCS) da Biblioteca Virtual em Saúde do Centro Latino-Americano e do Caribe de Informação em Ciências da Saúde (BVS/ Bireme). Essa revisão serviu para conhecer os principais documentos relativos à formação, bem como as análises sobre o tema realizadas pelos autores. Em seguida, para discutir de que forma a política de formação se expressou, mapearam-se cursos ativos de gestão em saúde, em nível de graduação, sequenciais e de pós-graduação lato e stricto sensu, e do ensino técnico a partir do levantamento em sítios institucionais. O universo analisado é de 639 cursos.
O levantamento foi realizado em três momentos: no mês de março de 2015, selecionaram-se 180 cursos de graduação, sequenciais e 297 cursos de especialização no Sistema de Regulação do Ensino Superior do Ministério da Educação (e-MEC). Para essa busca, utilizou-se os seguintes descritores: Gestão em Saúde; Gestão de Saúde; Gestão da Saúde; Gestão de Serviços de Saúde; Gestão Hospitalar; Gestão e Saúde; Gestão em Sistemas e Serviços de Saúde e Administração Hospitalar.

Em outubro de 2015, selecionaram-se dez cursos de mestrado e um de doutorado no Portal da Coordenadoria de Aperfeiçoamento de Pessoal de Nível Superior (Capes), nas áreas de conhecimento: Ciências da Saúde, Multidisciplinar e Ciências Sociais e Aplicadas.

Entre fevereiro e março de 2016, selecionaram-se 151 cursos de ensino técnico com a denominação de Gerência em Saúde, localizados no Sistema Nacional de Informações da Educação Profissional e Tecnológica do Ministério da Educação (Sistec).

Foram excluídos da seleção os cursos que não estavam ativos no momento do levantamento. Os dados foram organizados por nome do curso, região de oferta, modalidade, carga horária, categoria administrativa das instituições e ano de início. Para fins de apresentação e discussão dos resultados, foram elaboradas tabelas e gráficos.

O projeto de pesquisa foi aprovado pelo Comitê de Ética em Pesquisa da Escola Nacional de Saúde Pública Sergio Arouca, da Fundação Oswaldo Cruz (Ensp/Fiocruz), sob o número CAAE 52411416.9.0000.5240.

\section{Antecedentes da formação em gestão em saúde}

O aprimoramento da gestão dos serviços públicos, entre eles os serviços de saúde, tem sido preocupação em diferentes países. Gilles Dussault, em entrevista a Eleonor 
Conill, Ligia Giovanella e José Freire (2011), ressalta que existem diferenças entre os países de tradição anglo-saxônica e os países de cultura latina. Entre elas, encontra-se a tradição de formação em gestão em saúde, assim como o grau de profissionalização e a despolitização da gestão dos serviços de saúde, a seleção dos gestores de forma autônoma no nível das organizações e a menor rotatividade do pessoal dirigente quando há troca de ministro ou de diretor do hospital.

No Brasil, os conteúdos que poderiam ser compreendidos como de cunho administrativo, estavam presentes nos cursos de saúde pública oferecidos nas primeiras décadas do século XX, por meio do Departamento Nacional de Saúde. Para Kisil (1994), com a criação das Escolas de Saúde Pública, a partir de 1929, ano em que foi criada a Faculdade de Saúde Pública da Universidade de São Paulo (USP), introduziram-se na formação do sanitarista conteúdos de administração em saúde em uma grade curricular que previa a estatística sanitária, a epidemiologia, o saneamento do meio e a microbiologia. Tal modelo de formação teve como influência o ensino das Escolas de Saúde Pública dos Estados Unidos. O modelo americano foi também a base para a organização do primeiro curso de especialização em administração hospitalar, criado em 1951, em decorrência da necessidade de formar administradores quando da implantação do Hospital das Clínicas da USP.

Segundo o mesmo autor, um dos antecedentes da formação em gestão em saúde é o Programa de Coordenação e Apoio à Educação em Administração de Atenção à Saúde na América Latina e Caribe, de 1979, desenvolvido pela Organização PanAmericana de Saúde (Opas) com o apoio da Fundação W. K. Kellogg, e os Programas de Administração de Saúde.

Um aspecto relevante é que, na maioria das vezes, a procura pelos cursos na área partia de iniciativas individuais, como apontado por Ozslak (1995, P. 53):
A demanda de formação tende a se converter, deste modo, no resultado de uma atividade atomizada, onde prevalece a iniciativa individual dos funcionários envolvidos para além da necessidade institucional de contar com pessoal capacitado. Com poucas exceções, cada funcionário determina que conteúdos e orientações são relevantes para incrementar seus conhecimentos e tecnologias, buscando para si os provedores que possam satisfazer tais inquietudes.

Com a implementação do SUS, as iniciativas de formação em gestão em saúde, diferentemente das décadas anteriores, inseriram-se no contexto mais amplo da política de saúde, no qual se fazem presentes um conjunto de paradigmas, forças sociais e interesses, que às vezes são conflitantes e contraditórios ou convergentes entre si.

Mesmo tendo sido explicitado, por meio do art. 200. da Constituição Federal, o papel do setor saúde de ordenar a formação profissional, e que estabelece que compete ao SUS

III- ordenar a formação de Recursos Humanos na área da saúde; [...] $\vee$ - incrementar em sua área de atuação o desenvolvimento científico e tecnológico (BRASIL, 1988, P. 104-105),

a sua efetivação não foi imediata, e o mandato de regulamentar parte considerável da formação e capacitação permaneceu no Ministério da Educação. Embora o SUS represente um importante mercado de trabalho, apenas recentemente sua abordagem foi inserida no ensino da área, tanto no nível técnico quanto de graduação e de pós-graduação (BRASIL, 2003).

Nessa direção, cumpre ressaltar a criação de um conjunto de movimentos, seja de âmbito societal ou institucional, que se voltaram à articulação do ensino em saúde com os serviços como campo de práticas e a efetivação dos princípios e diretrizes do SUS. Dentre eles, cita-se a criação da Rede Unida, em 1997. Outros marcos históricos apontados por Lima et al. (2015), após os anos 2000, foram o Programa Nacional de Incentivo a Mudanças 
Curriculares nos Cursos de Medicina(Promed), os Polos de Capacitação em Saúde da Família e as Residências Multiprofissionais e a criação, em 2003, da Secretaria de Gestão do Trabalho e da Educação na Saúde (SGTES), no Ministério da Saúde (MS), que teve como o objetivo a definição de políticas e programas de formação profissional para o SUS. Essa Secretaria também foi responsável pela implementação da Política de Educação Permanente em Saúde (Peps), iniciada em 2007 e conduzida a partir de instâncias intersetoriais e interinstitucionais permanentes, denominadas Comissões Permanentes de Integração Ensino-Serviço (Cies). Outras iniciativas, voltadas à reorientação do perfil e da prática dos profissionais direcionando-as às necessidades de saúde da população ao fortalecimento e consolidação do SUS, foram o AprenderSUS, o ProSaúde, o PetSaúde e a ProResidência.

No que se refere às Conferências Nacionais de Saúde, as diferentes iniciativas para a oferta de cursos em gestão em saúde convergiram com os momentos da formulação da política. Dos trechos selecionados por Ceccim, Armani e Rocha (2002) dos relatórios finais, identificaram-se aqueles relativos à formação e desenvolvimento dos Recursos Humanos (RH) em saúde. Na X Conferência Nacional de Saúde, realizada em 1996:

[...] os Ministérios da Saúde e da Educação devem estimular a implementação de Programas de Especialização em Saúde Pública e em Gerenciamento de Serviços de Saúde desenvolvidos pelas universidades em parcerias com os governos Estaduais e/ou Municipais. (BRASIL, 1996 APUD CECCIM; ARMANI; ROCHA, 2002, P. 377).

Um dos cursos que se inserem nessa perspectiva é o Projeto Gerus - Desenvolvimento Gerencial de Unidades de Saúde do SUS. Desenvolvido em 1997 por meio do estabelecimento de parcerias entre as três esferas político-administrativas do SUS, universidades e a Opas, com a participação do Conselho das Secretarias Municipais de Saúde (Cosems), do
Conselho Nacional de Secretarias Municipais de Saúde (Conasems) e do Conselho Nacional de Secretários de Saúde (Conass), foi previsto como um curso de especialização, com carga horária mínima de 400 horas. Esse projeto voltou-se para a formação de gerentes de unidades de saúde com nível superior. Seu propósito era o de atender à demanda crescente de serviços de saúde de melhor qualidade, apoiar o processo de descentralização e se constituir como um instrumento de reorientação do modelo assistencial (BRASIL, 1997).

No ano 2000, realizou-se a XI Conferência Nacional de Saúde, e, no que diz respeito à Política Nacional de Recursos Humanos para o SUS, algumas das sugestões formuladas se referiam à formação de gestores de saúde:

- formar gerentes para o SUS com capacitação em administração pública, envolvendo gestores, assessores e dirigentes do SUS.

- garantir a realização de programas de capacitação de gerentes para a gestão de recursos humanos e elaboração de projetos técnicos quanto aos sistemas de informação e outros. (BRASIL, 2000 APUD CECCIM; ARMANI; ROCHA, 2002, P. 379).

Nos anos seguintes à XI Conferência, foram oferecidos dois outros cursos em consonância com as propostas apresentadas acima. O primeiro se refere ao Curso de Atualização em Gestão Municipal na Área da Saúde. De âmbito nacional, esse curso se voltou à capacitação de gestores municipais, tendo em vista o contexto de mudança do poder público produzido a partir das eleições realizadas no ano 2000. O desenho adotado visava ao aprofundamento das informações sobre o SUS no sentido de consolidação do processo de descentralização, bem como à troca de experiências, à reflexão sobre as políticas de saúde a partir do contexto político-normativo do SUS e do papel dos gestores. A avaliação desse curso possibilitou a construção de uma proposta metodológica por um grupo de pesquisadores 
vinculados ao Instituto de Medicina Social, da Universidade do Estado do Rio de Janeiro. Os pesquisadores chamaram a atenção para a importância da avaliação sistematizada dos processos de capacitação realizados nos serviços de saúde e da construção de uma cultura avaliativa (PIERANTONI ET AL., 2003).

O segundo foi instituído a partir do ano de 2006. Trata-se do Programa de Qualificação e Estruturação da Gestão do Trabalho e da Educação no SUS (ProgeSUS). Assim como os demais cursos mencionados anteriormente, foi proposto em parceria entre o MS, instituições acadêmicas, Conselhos Gestores e a Opas. Entre os seus objetivos, estava a qualificação de profissionais da área de gestão do trabalho e de gestão de informação no SUS para a implementação de modelos de gestão do trabalho comprometidos com as políticas de saúde (BRASIL, 2006).

A área de RH tem sido enfatizada nas ações do MS. No que se refere à formação, destacam-se outros dois cursos, que revelam os antecedentes e a importância desse componente: o Curso de Especialização em Gestão e Desenvolvimento de Recursos Humanos (CADRHU) e o Curso de Especialização em Gestão de Recursos Humanos, este voltado a 52 gerentes de $\mathrm{RH}$ das Secretarias de Estado de Saúde e órgãos centrais do MS (BRASIL, 2003).

Como perspectiva formativa, o mestrado profissional foi apontado como iniciativa fundamental para a formação em gestão, pensado especialmente para as esferas estadual e federal do SUS e para a "produção de conhecimento em áreas críticas da gestão central do SUS" (BRASIL, 2003, P. 16). Esse mestrado, que foi criado pela Capes no ano de 1995, partiu da constatação da necessidade de formar, além de docentes e pesquisadores, profissionais com perfis de alta qualificação e produtividade, capazes de refletir e atuar criticamente sobre o seu processo de trabalho. A previsão era de que a identificação de demandas por formação não deveria partir apenas dos indivíduos, mas sobretudo das instituições (HORTALE ET AL., 2010).
A importância do mestrado profissional para formação de RH estratégicos foi destacada por Goldbaum (2006). Em suas reflexões, esse autor aponta a adequação do mestrado profissional para a formação de quadros adaptados às novas necessidades do SUS. Não apenas pela capacidade de responder às necessidades específicas de cada um dos níveis de atuação, mas também, e com maior destaque, para a formação dos responsáveis pela definição e condução das grandes diretrizes técnicas dos órgãos do SUS. Este último aspecto guarda relação com a capacidade limitada de oferta de programas dessa natureza, exigindo a racionalidade da oferta e de forma a evitar descontinuidades e restrições na expansão de programas e projetos de outra natureza das instituições formadoras. É interessante observar que o primeiro curso de mestrado profissional foi realizado na área de administração em 1998, logo após sua normatização pela Capes. Já na área da saúde pública, a Escola Nacional de Saúde Pública, ofereceu, em 2002, ao MS o Curso de Gestão de Sistemas e Serviços de Saúde (ARTMANN, 2006).

Esses marcos históricos sobre a formação em gestão em saúde trouxeram elementos para a reflexão sobre a relação entre a formação de gestores e a implementação da política de saúde prevista no SUS. A seguir, serão discutidas as principais características da oferta dos cursos de gestão em saúde no Brasil, como resultado dessa política de formação e desenvolvimento dos RH em saúde.

\section{Principais características da oferta de cursos de gestão em saúde no Brasil}

\section{Diversidade e tendência dos cursos de gestão em saúde a partir de suas denominações}

Os cursos de gestão na área da saúde se caracterizam pela diversidade de denominações, principalmente no que se refere à 
educação de nível superior. Em algumas dessas denominações, entretanto, chama a atenção a semelhança entre os termos, com distinção apenas pelo uso de diferentes preposições. A tabela 1 apresenta a distribuição do conjunto desses cursos.

No caso dos cursos de especialização, as diferentes denominações podem indicar seu foco. Como exemplo, têm-se os cursos de 'gestão em saúde', que apresentavam denominações específicas: saúde coletiva, saúde ambiental, saúde mental, saúde coletiva indígena, saúde da família, saúde do trabalhador e segurança do trabalho, saúde da pessoa idosa, saúde na Amazônia, da clínica, do sistema prisional, ênfase em Perícia Médica, sistemas e serviços de saúde, qualidade, acreditação, tecnologia e inovação, informática, hospitalar, entre outros. Tais cursos, com início nos anos 2000, especialmente após o ano de 2010, poderiam indicar o caráter transversal da gestão e a preocupação de condução da política e das práticas de saúde em diferentes áreas.

Tabela 1. Distribuição por descritor de cursos de habilitação técnica, graduação e sequenciais, especialização, mestrado e doutorado em Gestão

\begin{tabular}{|c|c|c|c|c|c|c|c|c|c|c|c|}
\hline Descritor do curso & Habil. téc * & $\%$ & 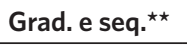 & $\%$ & Esp. ${ }^{\star \star}$ & $\%$ & $M^{\star \star \star}$ & $\%$ & $D^{\star \star \star}$ & $\%$ & Total \\
\hline Gestão em Saúde & - & & 1 & 0,5 & 106 & 35,7 & - & & - & & 107 \\
\hline Gestão em Saúde Ambiental & - & & 2 & 1 & - & & - & & - & & 2 \\
\hline Gestão em Saúde Coletiva & - & & 1 & 0,5 & - & & - & & - & & 1 \\
\hline $\begin{array}{l}\text { Gestão em Saúde Coletiva } \\
\text { Indígena }\end{array}$ & - & & 1 & 0,5 & - & & - & & - & & 1 \\
\hline Gestão de Saúde & - & & 2 & 1 & 33 & 11,1 & - & & - & & 35 \\
\hline Gestão da Saúde & - & & - & & 23 & 7,7 & - & & - & & 23 \\
\hline Gestão de Serviços de Saúde & - & & 3 & 1,7 & 16 & 5,4 & 2 & 20 & - & & 21 \\
\hline $\begin{array}{l}\text { Gestão de Sistemas e Serviços } \\
\text { de Saúde }\end{array}$ & - & & - & & 3 & 1 & - & & - & & 3 \\
\hline Gestão Hospitalar & - & & 168 & 93,3 & 67 & 22,5 & - & & - & & 235 \\
\hline Administração Hospitalar & - & & 2 & 1 & 49 & 16,5 & - & & - & & 51 \\
\hline Gerência em Saúde & 151 & 100 & - & & - & & - & & - & & 151 \\
\hline Gestão da Clínica & - & & - & & - & & 1 & 10 & - & & 1 \\
\hline $\begin{array}{l}\text { Gestão da Qualidade em Serviços } \\
\text { de Saúde }\end{array}$ & - & & - & & - & & 1 & 10 & - & & 1 \\
\hline Gestão de Organizações de saúde & - & & - & & - & & 1 & 10 & - & & 1 \\
\hline $\begin{array}{l}\text { Gestão de Programas e Serviços } \\
\text { de Saúde }\end{array}$ & - & & - & & - & & 1 & 10 & - & & 1 \\
\hline $\begin{array}{l}\text { Gestão de tecnologia e inovação } \\
\text { em saúde }\end{array}$ & - & & - & & - & & 1 & 10 & - & & 1 \\
\hline Políticas e Gestão em Saúde & - & & - & & - & & 1 & 10 & - & & 1 \\
\hline Gestão em Sistemas de Saúde & - & & - & & - & & 1 & 10 & - & & 1 \\
\hline Gestão e Informática em Saúde & - & & - & & - & & 1 & 10 & 1 & 100 & 2 \\
\hline TOTAL & 151 & 100 & 180 & 100 & 297 & 100 & 10 & 100 & 1 & 100 & 639 \\
\hline
\end{tabular}

Fonte: E-MEC, 2015; Plataforma Sucupira, 2015; Site Sistec, 2016.

* Habilitação Técnica: <http://www.sistec.mec.gov.br/>.

** Graduação e Sequenciais e Especialização: <http://emec.mec.gov.br/>.

${ }^{\star * \star *}$ Mestrado e Doutorado: <https://sucupira.capes.gov.br/sucupira/public/consultas/coleta/programa/listaPrograma.jsf;jsessionid=pLY6F5-eMDPG7PSJyQEuRv4B.sucupira-213>. 
O somatório dos cursos de graduação e sequenciais que incluem em sua denominação gestão hospitalar e administração hospitalar, quando comparados com o conjunto de cursos com outras denominações, permitiu identificar a predominância da formação em gestão voltada à área hospitalar. Eles representam 94,3\% do total dos cursos nesse nível de formação. Quanto aos cursos de especialização, observa-se uma mudança nesse percentual, mas ainda um direcionamento da formação para a área hospitalar, com $42,7 \%$ de cursos que associam os termos gestão ou administração e o termo hospitalar em sua denominação.

Na graduação e na especialização, o perfil de cursos predominantemente voltados para a área hospitalar indicaria a manutenção da ênfase nos hospitais como locus da atenção à saúde. Os cursos de gestão em saúde que se voltam a outras áreas como saúde da família, saúde mental, saúde ambiental, saúde coletiva tiveram início mais recentemente. Da graduação, citam-se: o Curso de Gestão em Saúde Coletiva, os dois cursos de Gestão Ambiental e o Curso de Gestão em Saúde Coletiva Indígena. Na especialização, só a partir de 2002 surgem cursos voltados à gestão da saúde ambiental e gestão da saúde da família. Apesar de poderem existir cursos com diferentes denominações e com oferta legal e regular, que não constem do Catálogo Nacional dos Cursos Superiores de Tecnologia, na consulta a ele, identificou-se apenas o curso 'Gestão Hospitalar' relacionado com a gestão em saúde (BRASIL, 2010).
Os cursos de formação técnica possuem a denominação de 'gerência em saúde' e são oferecidos por 85 instituições de ensino.

No mestrado, os dez cursos apresentam denominações relacionadas com: a clínica, os serviços de saúde, a qualidade em serviços, as organizações de saúde, os programas e serviços, a tecnologia e inovação, as políticas e gestão, os sistemas e informática. O único curso de doutorado encontrado está credenciado na área Interdisciplinar da Capes, com a denominação 'gestão e informática em saúde'.

\section{Concentração ou dispersão? Um panorama sobre a oferta de cursos de gestão em saúde nas regiões do Brasil}

A oferta de um mesmo curso pode se dar em mais de uma cidade ou estado, como nos casos dos cursos a distância. Ao se verificar a distribuição dessa oferta de cursos, constatou-se que há uma distribuição desigual nas diferentes regiões do Brasil (tabela 2). As Regiões Sudeste e Norte têm, respectivamente, o maior $(39,5 \%)$ e o menor $(10,9 \%)$ número de oferta de cursos em todos os níveis de formação. Na Região Nordeste, existem $25,5 \%$ dos cursos; na Região Sul, $12,8 \%$ e na Região Centro-Oeste, $11 \%$. A oferta de cursos de graduação e sequenciais, entretanto, é praticamente idêntica ao se considerar as Regiões Sudeste e Nordeste, bem como entre as Regiões Centro-Oeste, Sul e Norte. 
Tabela 2. Distribuição da oferta de cursos de gestão por região, descritor e nível de formação

\begin{tabular}{|c|c|c|c|c|c|c|c|c|}
\hline \multicolumn{2}{|c|}{ Cursos por região, descritor e nível de formação } & Habil. téc * & Grad. e seq. ${ }^{\star \star}$ & Esp. ${ }^{\star \star}$ & $M^{\star \star \star}$ & $D^{\star \star \star}$ & Total & $\%$ \\
\hline \multirow{2}{*}{ Sudeste } & gestão hospitalar & - & 69 & 66 & - & - & 135 & \multirow{2}{*}{39,5} \\
\hline & gestão saúde & 105 & 3 & 85 & 6 & 1 & 200 & \\
\hline \multirow{2}{*}{ Nordeste } & gestão hospitalar & - & 87 & 48 & & - & 135 & \multirow{2}{*}{25,5} \\
\hline & gestão saúde & 29 & 3 & 47 & 2 & - & 81 & \\
\hline \multirow{2}{*}{ Sul } & gestão hospitalar & - & 44 & 25 & - & - & 69 & \multirow{2}{*}{12,8} \\
\hline & gestão saúde & 10 & 1 & 28 & 1 & - & 40 & \\
\hline \multirow{2}{*}{ Centro-Oeste } & gestão hospitalar & - & 40 & 18 & - & - & 58 & \multirow{2}{*}{11} \\
\hline & gestão saúde & 9 & 2 & 25 & - & - & 36 & \\
\hline \multirow{2}{*}{ Norte } & gestão hospitalar & - & 37 & 25 & - & - & 62 & \multirow{2}{*}{10,9} \\
\hline & gestão saúde & 2 & 2 & 26 & 1 & - & 31 & \\
\hline \multicolumn{2}{|l|}{ TOTAL } & 155 & 288 & 393 & 10 & 1 & 847 & 100 \\
\hline
\end{tabular}

Fonte: E-MEC, 2015; Plataforma Sucupira, 2015; Site Sistec, 2016

* Habilitação Técnica: <http://www.sistec.mec.gov.br/>.

** Graduação e Sequenciais e Especialização: <http://emec.mec.gov.br/>.

*** Mestrado e Doutorado: <https://sucupira.capes.gov.br/sucupira/public/consultas/coleta/programa/listaPrograma.jsf;jessionid=pLY6F5-eMDPG7PSJyQEuRv4B.sucupira-213>.

Tal fato confirma o estudo de Haddad et al. (2008) sobre a formação em saúde no Brasil, que se caracteriza por importantes desequilíbrios seja na formação e distribuição de profissionais, seja na oferta dos cursos técnicos de graduação e pós-graduação em saúde.

A maior oferta de cursos de gestão em saúde na região Sudeste guarda relação com o número expressivo de instituições formadoras nessa mesma região. A análise do predomínio dos cursos por região, entretanto, deverá ser refinada ao se considerar o cruzamento dessa oferta formativa com a estruturação institucional do setor saúde e a existência de serviços de saúde.

\section{Os cursos de gestão em saúde e as modalidades presencial e a distância}

Os cursos presenciais predominam em relação aos cursos a distância (EAD), tanto nos cursos de habilitação técnica (97,3\%), de graduação e sequenciais (94,5\%), quanto nos cursos em nível de especialização (91,6\%). Os cursos de mestrado e doutorado são oferecidos somente na modalidade presencial (tabela 3).

Tabela 3. Distribuição dos cursos de gestão por modalidade

\begin{tabular}{|c|c|c|c|c|c|c|c|c|c|c|c|}
\hline Nível de formação / modalidade & Habil. téc * & $\%$ & Grad. e seq. ${ }^{\star \star}$ & $\%$ & Esp. ${ }^{\star \star}$ & $\%$ & $M^{\star \star \star}$ & $\%$ & $D^{\star \star \star}$ & $\%$ & Total \\
\hline Presencial & 147 & 97,3 & 170 & 94,5 & 250 & 91,6 & 10 & 100 & 1 & 100 & 578 \\
\hline EAD & 4 & 2,7 & 10 & 5,5 & 47 & 8,4 & - & & - & & 61 \\
\hline TOTAL & 151 & 100 & 180 & 100 & 297 & 100 & 10 & 100 & 1 & 100 & 639 \\
\hline
\end{tabular}

Fonte: E-MEC, 2015; Plataforma Sucupira, 2015; Site Sistec, 2016.

* Habilitação Técnica: <http://www.sistec.mec.gov.br/>.

** Graduação e Sequenciais e Especialização: <http://emec.mec.gov.br/>.

${ }^{\star \star \star \star}$ Mestrado e Doutorado: <https://sucupira.capes.gov.br/sucupira/public/consultas/coleta/programa/listaPrograma.jsf;jessionid=pLY6F5-eMDPG7PSJyQEuRv4B.sucupira-213>. 
Em nível de graduação, dos dez cursos nessa modalidade, somente um era oferecido por instituição pública. Dos dados disponíveis no Sistec, encontraram-se quatro cursos de habilitação técnica em gerência em saúde na modalidade EAD. Destes, metade são oferecidos por instituições privadas.

\section{Predomínio do privado sobre o públi- co na formação em gestão em saúde}

Constatou-se o expressivo número de instituições privadas (tabela 4). Estas sobressaem na oferta de cursos nos níveis de especialização (88,3\%), de graduação $(83,6 \%)$ e de habilitação técnica $(57,5 \%)$. Esse predomínio do setor privado de ensino se altera nos cursos de mestrado, com participação de $70 \%$ de instituições públicas. Os cursos de doutorado são oferecidos somente por instituições públicas. Em um olhar mais detalhado sobre a modalidade EAD, destaca-se que, dos 47 cursos ofertados em nível de especialização, 55,3\% eram oferecidos por instituições privadas.

Tabela 4. Distribuição dos cursos por nível de formação e categoria administrativa das instituições

\begin{tabular}{|c|c|c|c|c|c|c|c|c|c|c|c|c|}
\hline \multicolumn{2}{|c|}{$\begin{array}{l}\text { Cursos por nível de formação e categoria } \\
\text { administrativa da instituição }\end{array}$} & \multirow{2}{*}{$\begin{array}{r}\text { Habil. } \\
\text { téc * } \\
28\end{array}$} & \multirow{2}{*}{$\begin{array}{r}\% \\
18,5\end{array}$} & \multirow{2}{*}{$\begin{array}{r}\begin{array}{r}\text { Grad. e } \\
\text { seq. }^{\star \star}\end{array} \\
75\end{array}$} & \multirow{2}{*}{$\begin{array}{r}\% \\
41,6\end{array}$} & \multirow{2}{*}{$\frac{\text { Esp. * }^{\star \star}}{103}$} & \multirow{2}{*}{$\begin{array}{r}\% \\
34,8\end{array}$} & \multirow{2}{*}{$\frac{M^{\star \star \star}}{3}$} & \multirow{2}{*}{$\begin{array}{c}\% \\
30\end{array}$} & \multirow{2}{*}{$\frac{D^{\star \star \star}}{-}$} & \multirow[t]{2}{*}{$\%$} & \multirow{2}{*}{$\begin{array}{r}\text { Total } \\
209\end{array}$} \\
\hline Privada & sem fins lucrativos & & & & & & & & & & & \\
\hline & com fins lucrativos & 59 & 39 & 76 & 42 & 159 & 53,5 & - & & - & & 294 \\
\hline & 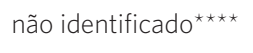 & 28 & 18,5 & - & & - & & - & & - & & 28 \\
\hline Subtotal & & 115 & 76 & 151 & 83,6 & 262 & 88,3 & 3 & 30 & 0 & 0 & 531 \\
\hline \multirow[t]{3}{*}{ Pública } & Federal & 15 & 10 & 20 & 11 & 24 & 8 & 3 & 30 & 1 & 100 & 63 \\
\hline & Estadual & 21 & 14 & - & & 9 & 3 & 4 & 40 & - & & 34 \\
\hline & Municipal & - & & 1 & 1 & 2 & 0,7 & - & & - & & 3 \\
\hline \multicolumn{2}{|c|}{ não informado } & - & & 8 & 4,4 & - & & - & & - & & 8 \\
\hline \multicolumn{2}{|l|}{ Subtotal } & 36 & 24 & 29 & 16,4 & 45 & 11,7 & 7 & 70 & 1 & 100 & 108 \\
\hline \multicolumn{2}{|l|}{ TOTAL } & 151 & 100 & 180 & 100 & 297 & 100 & 10 & 100 & 1 & 100 & 639 \\
\hline
\end{tabular}

Fonte: E-MEC, 2015; Plataforma Sucupira, 2015; Site Sistec, 2016.

* Habilitação Técnica: <http://www.sistec.mec.gov.br/>.

** Graduação e Sequenciais e Especialização: <http://emec.mec.gov.br/>

*** Mestrado e Doutorado: <https://sucupira.capes.gov.br/sucupira/public/consultas/coleta/programa/listaPrograma.jsf;jsessionid=pLY6F5-eMDPG7PSJyQEuRv4B.sucupira-213>.

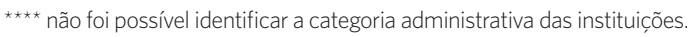

O panorama da forte presença do setor privado no ensino superior foi estudado por Santos e Guimarães-Iosif (2012). Para as autoras, a educação sofre a influência do modelo de gestão presente no neoliberalismo. Em decorrência, nas últimas décadas, a educação passa a ser alvo de interesses mercadológicos por parte de diferentes atores nacionais e internacionais, fruto de um modelo de governança que tem como diretrizes a privatização, a desregulação estatal e a redefinição das políticas públicas conforme os interesses do mercado.

\section{Início dos cursos de gestão em saúde}

Ao se levar em consideração o ano de início do funcionamento dos cursos, observou-se que, na graduação, o mais antigo na modalidade presencial é o Curso de Administração 
Hospitalar, da Faculdade de Educação Superior do Paraná (FESPPR), com início em 1983 (tabela 5). Os demais cursos de graduação e sequenciais foram oferecidos a partir dos anos 2000. Ademais, a partir do ano de 2004, o número de cursos de graduação e sequenciais iniciados a cada ano foi crescente até 2009, ano com maior número de cursos iniciados. Entre os cursos com início em 2009, encontra-se o bacharelado em Gestão de Serviços de Saúde, ofertado pela Universidade Federal de Minas Gerais (UFMG). Segundo Faria e Silva (2016), esse curso surge no contexto do Programa de Apoio ao Plano de Reestruturação e Expansão das Universidades Federais (Reuni), do Ministério da Educação, com a premissa de antecipar a formação dos gestores do sistema e dos serviços de saúde da pós-graduação para o nível de graduação.

No que diz respeito ao período de oferta dos cursos técnicos, apenas 15 cursos começaram entre 2012 e 2015. No entanto, devem-se ponderar esses dados dentro de seus limites, já que em $60 \%$ dos cursos esse dado não foi informado.

Observou-se um considerável crescimento de cursos de gestão em saúde nos últimos 15 anos, especialmente a partir dos anos 2002. A criação da SGTES pelo MS poderia guardar relação com essa realidade e como busca de enfrentamento do desafio da gestão do SUS, a partir da iniciativa de formação de seus gestores.

Tabela 5. Distribuição dos cursos por ano de início e nível de formação

\begin{tabular}{|c|c|c|c|c|c|c|c|c|c|c|c|}
\hline $\begin{array}{l}\text { Nível de formação / ano de início } \\
\text { do curso }\end{array}$ & Habil. téc * & $\%$ & Grad. e seq. ${ }^{\star \star}$ & $\%$ & Esp. ${ }^{\star \star}$ & $\%$ & $M^{\star \star \star}$ & $\%$ & $D^{\star \star \star}$ & $\%$ & Total \\
\hline 1983 & - & & 1 & 0,5 & - & & - & & - & & 1 \\
\hline 1995-2001 & - & & - & & 2 & 0,7 & - & & - & & 2 \\
\hline $2002-2006$ & - & & 17 & 9,4 & 21 & 7 & - & & - & & 38 \\
\hline 2007-2011 & 45 & 29,8 & 96 & 53,3 & 65 & 21,9 & 1 & 10 & - & & 207 \\
\hline $2012-2015$ & 15 & 10 & 35 & 19,4 & 201 & 67,7 & 6 & 60 & 1 & 100 & 258 \\
\hline não informado & 91 & 60,2 & 31 & 17,2 & 8 & 2,7 & 3 & 30 & - & & 133 \\
\hline TOTAL & 151 & 100 & 180 & 100 & 297 & 100 & 10 & 100 & 1 & 100 & 639 \\
\hline
\end{tabular}

Fonte: E-MEC, 2015; Plataforma Sucupira, 2015; Site Sistec, 2016.

* Habilitação Técnica: <http://www.sistec.mec.gov.br/>.

** Graduação e Sequenciais e Especialização: <http://emec.mec.gov.br/>.

${ }^{\star * \star}$ Mestrado e Doutorado: <https://sucupira.capes.gov.br/sucupira/public/consultas/coleta/programa/listaPrograma.jsf;jsessionid=pLY6F5-eMDPG7PSJyQEuRv4B.sucupira-213>.

\section{Avanços, desafios e perspectivas da formação em gestão em saúde}

Inúmeras conquistas foram alcançadas em relação à formação em gestão em saúde no Brasil. Alguns desses avanços foram apreendidos a partir do estudo realizado. Entre eles, estão a oferta nacional de cursos em consonância com a política de saúde prevista no SUS, a concretização de diferentes cursos realizados por meio de parcerias interinstitucionais, iniciativas formativas realizadas no contexto do Programa Nacional de Capacitação de Gestores Municipais, a intensificação da oferta de cursos de graduação 
nas universidades federais com o Reuni, a formação de significativo contingente de ocupantes de cargos dirigentes, fortalecendo a gestão descentralizada, a perspectiva da importância da avaliação de projetos de capacitação com apontamentos para o seu aprimoramento, a oferta crescente de cursos voltados à gestão em diferentes áreas, como os sistemas e serviços de saúde, saúde da família, saúde mental, saúde ambiental, auditoria, entre outros, ampliando a oferta para além da área hospitalar.

Um conjunto de desafios, entretanto, permanece. Quanto aos cursos de pós-graduação stricto sensu, verificou-se que predominam os cursos de mestrado profissionais, com nove cursos. Apesar desse nível de formação ter sido apontado como iniciativa fundamental na Política de Recursos Humanos para o SUS, pensado especialmente para os níveis estadual e federal do SUS (BRASIL, 2003), a oferta identificada por meio do levantamento sugere uma concentração e restrição de vagas quando comparada com o contingente potencial de gestores do SUS. Tal desafio nesse nível de ensino remete, ainda, a dificuldades relativas à existência de docentes e pesquisadores especializados na área da gestão em saúde.

No que se refere à formação técnica, chama a atenção que dos 151 cursos encontrados, apenas $23,8 \%$ são oferecidos por instituições públicas e, destes, apenas 1,9\% são ofertados pelas Escolas Técnicas do SUS, que são a Escola Politécnica de Saúde Joaquim Venâncio (EPSJV/Fiocruz) e a Escola Técnica de Saúde do Centro de Educação Profissional e Tecnológica da Universidade Estadual de Montes Claros (ETS/CEPT/Unimontes).

No ano de 2009, o MS lançou o Programa de Formação de Profissionais de Nível Médio para a Saúde (Profaps), que prevê oito áreas técnicas estratégicas prioritárias para a educação profissional técnica de nível médio na saúde. A área de gestão em saúde não está incluída, apesar do expressivo contingente de trabalhadores que atuam nela, conforme os dados do Cadastro Nacional de Estabelecimentos de Saúde (CNES/ Datasus), de julho de 2016, que apontam que somente as ocupações de assistente técnico administrativo e técnico em administração já correspondem a um total de 68.156 profissionais. Existem, entretanto, muitas outras ocupações administrativas de nível médio cujos profissionais assumem funções em todos os níveis do sistema de saúde. A não priorização da área de gestão em saúde pelo Profaps impacta de forma negativa na manutenção da insuficiência da oferta dessa área formativa para a qualificação do conjunto dos trabalhadores que atuam ou venham a atuar no SUS. Outro fator que pode caracterizar a pouca ênfase dessa formação é a não exigência de capacitação prévia para o ingresso nos postos de trabalho na área administrativa do SUS.

Outros desafios referem-se à concentração dos cursos e aos que, em sua quase totalidade, tiveram início nos últimos 15 anos, podendo caracterizar a baixa tradição de formação na área, o predomínio da oferta por instituições privadas e os voltados, nos cursos de graduação, quase que exclusivamente para a área de gestão hospitalar. Tais cursos de graduação voltados à gestão hospitalar são, na maioria, tecnológicos, na modalidade presencial (94\%) e oferecidos por instituições privadas (98\%).

Identificou-se, ainda, nos cursos de graduação, uma distinção do grau acadêmico, que é o título conferido pela conclusão com sucesso de um curso, ciclo ou etapa de estudos superiores, na área hospitalar das demais áreas de gestão em saúde. Verificouse que a distribuição dos cursos apresentou uma significativa diferença ao se tomar separadamente os cursos com denominações de gestão em saúde; gestão de saúde e gestão de sistemas de saúde dos cursos denominados de gestão hospitalar e de administração hospitalar. Nos primeiros, dos 10 cursos oferecidos (bacharelado, tecnológico, sequencial de formação específica e sequencial), 70\% deles é bacharelado. $\mathrm{O}$ mesmo não acontece nos 
cursos de graduação em gestão hospitalar e de administração hospitalar, em que predominam os cursos tecnológicos, ou seja, dos 170 cursos estudados, 93\% são tecnológicos, $5 \%$ sequenciais, $1 \%$ é sequencial de formação específica e $1 \%$ é de bacharelado.

A diversidade dos cursos de gestão em saúde também é evidenciada a partir da carga horária dos cursos de graduação e especialização. Na graduação, predominam os cursos com carga horária entre 2.400 e 3.300 horas. Nos cursos sequenciais, preconiza-se a carga horária mínima de 1.600 horas, conforme o Parecer CES (Câmara de Educação Superior) 968/98, do Conselho Nacional de Educação. Todos cursos sequenciais de gestão em saúde encontrados atendem a essa determinação.

Dos 297 cursos de especialização, 38\% possuem a carga horária mínima preconizada de 360 horas. Estes são em sua quase totalidade oferecidos por instituições privadas e com fins lucrativos. Por outro lado, encontraram-se 22,5\% cursos com carga horária com mais de 450 horas. Destas, 73,1\% são instituições privadas em que 59,1\% têm fins lucrativos.

Apesar dos antecedentes históricos revelarem uma crescente busca pela integração e ampliação da institucionalidade da formação em gestão em saúde, a inexistência de uma política nacional bem definida e a não exigência de formação na área para ocupação de cargos dirigentes e em áreas administrativas do SUS impõem limites à efetivação dessa institucionalidade. A transformação desse cenário encontra-se na base das perspectivas futuras de ampliação e consolidação da formação em gestão em saúde, direcionando-a para uma gestão pública, de qualidade e em consonância com os princípios do SUS.

\section{Considerações finais}

Com a revisão, mesmo que não exaustiva, da produção teórica e empírica relacionada com o tema da formação em gestão em saúde, pôde-se compreender sua inserção em um campo mais amplo de discussão e investigação no contexto das políticas de saúde e de educação no Brasil. Caberia investigar como essa formação estaria condicionada por uma lógica política e econômica predominantemente neoliberal e influenciada por instituições multilaterais e organismos internacionais.

Com o breve histórico que foi apresentado, fica evidente que o aprimoramento dos serviços públicos de saúde, necessário à implementação do SUS, pressupõe a formação dos gestores de saúde. Considera-se, entretanto, que ainda que a educação seja tomada como fundamental no processo de qualificação dos serviços públicos de saúde e que a gestão seja vista como uma das variáveis centrais e um dos grandes desafios do SUS, a formação em gestão em saúde ainda não é uma política consolidada em nosso meio.

$\mathrm{Na}$ realização do estudo, encontraram-se algumas limitações. A falta de dados sobre os cursos de habilitação técnica em 'gerência em saúde' se constituiu em uma limitação do estudo. Em relação ao tipo de oferta (subsequente, concomitante ou integrado) e ano de início, em $62,9 \%$ e $60 \%$ dos cursos, respectivamente, esse dado não estava disponível no Sistec. Outra limitação encontrada diz respeito ao fato de que o número de cursos de graduação e de especialização pode ter sido subdimensionado ao se utilizar como fonte documental apenas o e-MEC, considerando que as iniciativas do MS que se voltam para cursos de capacitação não estão ali registradas. Nesse sentido, caberia realizar levantamento futuro de cursos oferecidos por instituições como a Fiocruz, a Universidade Aberta do Brasil, o MS, entre outros, que não constam no e-MEC.

Estudos futuros podem aprofundar as concepções de formação presentes nos espaços de formulação da política de saúde e seus desdobramentos na oferta de cursos de gestão em saúde. Caberia, igualmente, aprofundar a reflexão se o atual perfil dos cursos de gestão, dado o seu predomínio na área hospitalar, especialmente na graduação, e 
oferecidos por instituições privadas; impactam na substituição do modelo de atenção centrado na recuperação da saúde e no espaço hospitalar em direção ao modelo de atenção universal, integral e equânime pretendido pelo SUS.

\section{Colaboradores}

Maria Luiza Silva Cunha e Virginia Alonso Hortale participaram igualmente de todas as etapas de elaboração do artigo.

$\mathrm{O}$ artigo é resultado parcial de pesquisa de doutoramento da autora principal, sob a orientação da segunda autora.

\section{Referências}

ARTMANN, E. Desafios na formação de recursos humanos para o SUS: a experiência do Mestrado Profissionalizante da Ensp com a SAS-MS. In: LEAL, M. C.; FREITAS, C. M. (Org.). Cenários possíveis: experiências e desafios do mestrado profissional na saúde coletiva. Rio de Janeiro: Fiocruz, 2006. p. 151-189.

BRASIL. Constituição (1988). Constituição da República Federativa do Brasil. Brasília, DF: Senado Federal, 1988. Disponível em: <http://www2.camara.leg.br/atividade-legislativa/legislacao/Constituicoes_Brasileiras/constituicao1988.html/ConstituicaoTextoAtualizado_EC96. pdf $>$. Acesso em: 20 jun. 2017.

Ministério da Educação. Catálogo Nacional de Cursos Superiores de Tecnologia 2010. Brasília, DF: Ministério da Educação, 2010. Disponível em: $<$ http://portal.mec.gov.br/index.php?option=com docman\&view=download\&alias=13029-catalogo-

\section{Agradecimentos}

À Prof. ${ }^{a}$ Dr. ${ }^{a}$ Marly Marques da Cruz, do Departamento de Administração e Planejamento em Saúde (Daps), da Escola Nacional de Saúde Pública Sergio Arouca, da Fundação Oswaldo Cruz (Ensp/Fiocruz), pela leitura e orientação na oficina de artigos, promovida pelo Programa de Pós-Graduação em Saúde Pública da Ensp/Fiocruz, realizada em abril de 2016.

À Hélen Patrícia Ferreira de Oliveira, bolsista do Programa Institucional de Bolsas de Iniciação Científica (Pibic/Fiocruz), pelo levantamento dos cursos de habilitação técnica em gerência em saúde.

-nacional-cursos-superiores-tecnologia-2010-

-290413-pdf\&category_slug=abril-2013-

pdf\&Itemid=30192>. Acesso em: 13 out. 2015.

Ministério da Saúde. Secretaria de Gestão do

Trabalho e da Educação na Saúde. Departamento de Gestão e da Regulação do Trabalho em Saúde. Programa de Qualificação e Estruturação da Gestão do Trabalho e da Educação no SUS. Brasília, DF: Ministério da Saúde, 2006. Disponível em: <http://bvsms.saude. gov.br/bvs/publicacoes/cartilhaprogesus.pdf>. Acesso em: 27 ago. 2013.

Ministério da Saúde. Secretaria de Políticas de Saúde. Coordenação-Geral da Política de Recursos Humanos. Política de Recursos Humanos para o SUS: balanço e perspectivas. Brasília, DF: Ministério da Saúde, 2003. 
. Ministério da Saúde. Secretaria de

Projetos Especiais de Saúde. Coordenação Geral de

Desenvolvimento de Recursos Humanos para o SUS.

Projeto Gerus: desenvolvimento Gerencial de Unidades de Saúde do SUS. Brasília, DF: Ministério da Saúde, 1997. Disponível em: <http://bvsms.saude.gov.br/bvs/ folder/10006002808.pdf>. Acesso em: 27 ago. 2013.

CAMPOS, G. W. S. A saúde pública e a defesa da vida. 3. ed. São Paulo: Hucitec, 2006.

CECCIM, R. B.; ARMANI, T.; ROCHA, C. O que dizem a legislação e o controle social em saúde sobre a formação de recursos humanos e o papel dos gestores públicos, no Brasil. Ciência \&t Saúde Coletiva, Rio de Janeiro, v. 7, n. 2, p. 373-383, jan. 2002.

\section{CONILL, E. M.; GIOVANELLA, L.; FREIRE, J. M.}

Entrevista com o professor Gilles Dussault: Desafios dos sistemas de saúde contemporâneos, por Eleonor Minho Conill, Ligia Giovanella e José-Manuel Freire. Ciência \&t Saúde Coletiva, Rio de Janeiro, v. 16, n. 6, p. 2889- 2892, jun. 2011.

E-MEC. 2015. Disponível em: <http://emec.mec.gov. br/>. Acesso em: 5 mar. 2015.

FARIA, M. A.; SILVA, A. J. Gestão de serviços de saúde: analisando a identidade na graduação. Saúde em Debate, Rio de Janeiro, v. 40, n. 108, p. 95-105, mar. 2016.

GOLDBAUM, M. Mestrado Profissionalizante em Saúde Coletiva. In: LEAL, M. C.; FREITAS, C. M. (Org.). Cenários possíveis: experiências e desafios do mestrado profissional na saúde coletiva. Rio de Janeiro: Fiocruz, 2006. p. 27-32.

HADDAD, A. E. et al. Política Nacional de Educação na Saúde. Revista Baiana de Saúde Pública, Salvador, v. 32. supl.1, p. 98-114, out. 2008.

HORTALE, V. A. et al. Características e limites do mestrado profissional na área da Saúde: estudo com egressos da Fundação Oswaldo Cruz. Ciência \&t Saúde Coletiva, Rio de Janeiro, v. 15, n. 4, p. 2051-2058, jul. 2010.
KISIL, M. Educação em Administração de Saúde na América Latina: a busca de um paradigma. São Paulo: USP, 1994.

LIMA, V. V. et al. Ativadores de processos de mudança: uma proposta orientada à transformação das práticas educacionais e da formação de profissionais de saúde. Ciência \&t Saúde Coletiva, Rio de Janeiro, v. 20, n. 1, p. 279-288, jan. 2015.

MACHADO, C. V.; LIMA, L. D; BAPTISTA, T. W. F. Configuração Institucional e o Papel dos Gestores no Sistema Único de Saúde. In: MATTA, G. C.; PONTES, A. L. P. Política de saúde: organização e operacionalização do Sistema Único de Saúde. Rio de Janeiro: EPSJV; Fiocruz, 2007. p. 139-162.

OZSLAK, O. As demandas de formação de administradores públicos frente ao novo perfil do Estado. Revista do Serviço Público, Brasília, DF, v. 119, n. 1, p. 45-67, jan./ abr. 1995.

PIERANTONI, C. R. Formação de gestores para o sistema de saúde. A experiência do mestrado profissional do Instituto de Medicina Social da UERJ. In: LEAL, M. C.; FREITAS, C. M. (Org.). Cenários possíveis: experiências e desafios do mestrado profissional na saúde coletiva. Rio de Janeiro: Fiocruz, 2006. p. 123-136.

PIERANTONI, C. R. et al. Avaliação do curso de atualização em gestão municipal na área da saúde: uma proposta metodológica. In: BRASIL. Ministério da Saúde. Observatório de recursos humanos em saúde no Brasil: estudos e análises. Rio de Janeiro: Fiocruz, 2003. p. 1-29.

PLATAFORMA SUCUPIRA. 2015. Disponível em: $<$ https://sucupira.capes.gov.br/sucupira/public/consultas/ coleta/programa/listaPrograma.jsf;jsessionid=pLY6F5-eMDPG7PSJyQEuRv4B.sucupira-213>. Acesso em: 15 out. 2015.

RODRIGUES, P. H. A. Desafios políticos para a consolidação do Sistema Único de Saúde: uma abordagem histórica. História, Ciências, Saúde - Manguinhos, Rio de Janeiro, v. 21, n. 1, p. 37-59, jan./mar. 2014. 
SANTOS, A. V.; GUIMARÃES-IOSIF, R. Política e Governança na Educação Superior no Brasil: mercantilização e comprometimento da qualidade. 2012. Disponível em: <http://www.anpae.org.br/seminario/ ANPAE2012/1comunicacao/Eixo01_19/Aline\%20 Veiga\%20dos\%20Santos_int_GTl.pdf >. Acesso em: 10 jul. 2016

SILVA, A. L. A. Educação profissional e gestão em saúde: pressupostos teórico-conceituais na formação de profissionais de nível médio. Trabalho Educação e Saúde, Rio de Janeiro, v. 3, n. 2, p. 351-370, set. 2005.
SITE SISTEC. 2016. Disponível em: <http://www. sistec.mec.gov.br/>. Acesso em: 10 fev. 2016.

VECINA NETO, G. A formação de administradores para o setor saúde: algumas considerações sobre o atual momento brasileiro e a responsabilidade da universidade. Revista de Administração Pública, Rio de Janeiro, v. 24, n. 4, p. 95-9, ago./out. 1990.

Recebido para publicação em setembro de 2016

Versão final em maio de 2017

Conflito de interesses: inexistente

Suporte financeiro: não houve 